Purdue University Purdue e-Pubs

ECE Technical Reports

Electrical and Computer Engineering

4-1-1995

Differential Current Switch Logic: A Low Power DCVS Logic Family

Dinesh Somasekhar

Purdue University School of Electrical Engineering

Kaushik Roy

Purdue University School of Electrical Engineering

Follow this and additional works at: http://docs.lib.purdue.edu/ecetr

Part of the Power and Energy Commons

Somasekhar, Dinesh and Roy, Kaushik, "Differential Current Switch Logic: A Low Power DCVS Logic Family" (1995). ECE Technical Reports. Paper 118.

http://docs.lib.purdue.edu/ecetr/118

This document has been made available through Purdue e-Pubs, a service of the Purdue University Libraries. Please contact epubs@purdue.edu for additional information. 


\section{DiFFERENTIAL CURRENT SWITCH LOGIC: A LOW POWER DCVS LOGIC FAMILY}

DINESH SOMASEKHAR

KAUSHIK ROY

TR-EE 95-11

APRIL 1995

SCHOOL OF ELECTRICAL ENGINEERING PURDUE UNIVERSTTY

West LAFAYETTE, INDIANA 47907-1285 


\title{
Differential Current Switch Logic: A Low Power DCVS Logic Family
}

\author{
Dinesh Somasekhar and Kaushik Roy \\ Electrical Engineering \\ Purdue University \\ West Lafayette, IN 47907
}

\begin{abstract}
We present a new logic family, Differential Current Switch Logic (DCSL) for implementing clocked CMOS circuits. The circuit is in principle a differential cascode voltage switch logic circuit (DCVS). In comparison to other forms of' clocked DCVS, DCSL achieves better performance both in terms of power and speed by restricting internal voltage swings in the $\mathrm{N}$ tree. Automatic lock-out of inputs on completion of evaluation is a novel feature of the circuit and allows new implementation of logic functions and the possibility of operating with reduced voltage swings. SPICE simulations carried out with. the MOSIS $1.2 \mu$ process indicate that DCSL is better than similar clocked DCVS circuits by a factor of two both in terms of power and speed, for moderate tree heights.
\end{abstract}




\title{
Differential Current Switch Logic: A Low Power DCVS Logic Family*
}

\author{
Dinesh Somasekhar and Kaushik Roy \\ Electrical Engineering \\ Purdue University \\ West Lafayette, IN 47907-1285, USA
}

\begin{abstract}
We presenl a new logic family, Differential Current Switch Logic (DCSL) for implementing clocked CMOS circuits. The circuit is in principle a differential cascode voltage swiich logic circuit (DCVS). In comparison lo other forms of clocked DCVS, DCSL achieves better performance bolh in terms of power and speed by restricting internal voltage swings in lhe $N$ tree. Automatic lock-out of inpuls on completion of evalualion is a novel feature of lhe circuii and allows new implementation of logic funclions and the possibilily of operaling wilh reduced voltage swings. SPICE simulations camed out wilh lhe MOSIS $1.2 \mu$ process indicate that DCSL is better lhan similar clocked DCVS circuits by a factor of two both in terms of power and speed, for moderate iree heighis.
\end{abstract}

\section{Introduction}

In the quest for achieving low-power, circuit design techniques have largely overlooked differential cascode logic circuits (DCVS) in favor of traditional CMOS styles. The high activity of DCVS gates and the need to route differential signals cause them to compare unfavorably with respect to implementations using static CMOS, complementary pass transistor logic, and differential pass transistor logic [1, 2]. However DCVS gates do offer the potential of having high fan-in which leads to a reduction in logic depth, high speed, and the capability of generating completion signals for asynchronous operations.

This paper presents a new clocked DCVS logic family called Differential Current Switch Logic $(D C S L)$. The salient features of this logic family are: sensing of differential currents

"The research was sponsored by IBM and ARPA under contract F33615-95-C-1625 


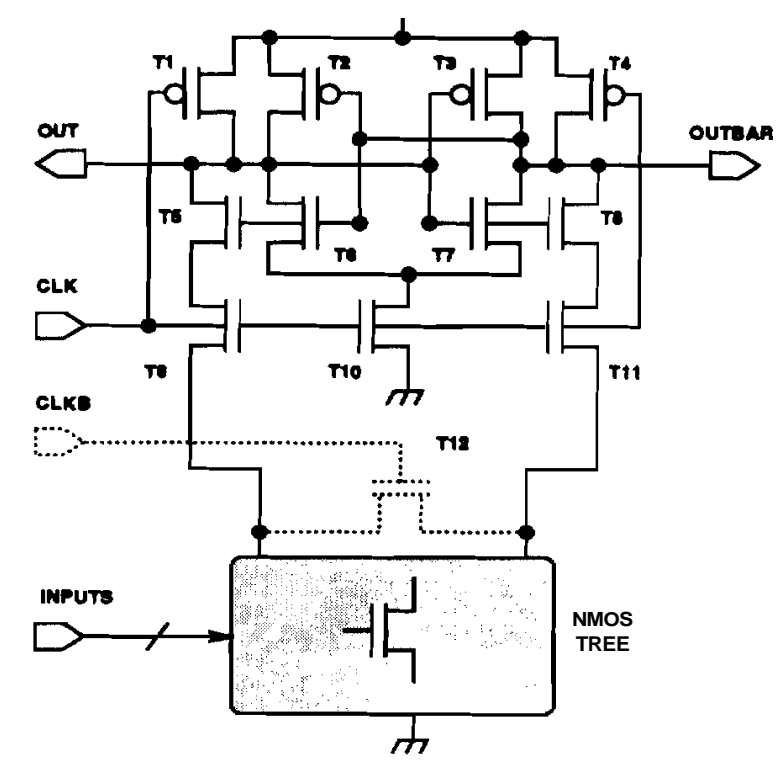

Figure 1: Precharge high DCSL

in an evaluation NMOS transistor tree, a very low voltage swing in internal nodes of the NMOS transistor tree, and an automatic lock-out of inputs once gate evaluation is complete. These features directly translate into high fan-in gates, low power consumption, and the possibility of implementing logic functions in new ways. We first describe the DCSL gate and its operation. The new implementation of logic functions, and the possibility of operating the gate with reduced voltage swings are illustrated next. SPICE simulations using the MOSIS $1.2 \mu$ process indicate the advantages of this gate over similar clocked DCVS gates which do not restrict the voltage swing of internal nodes.

\section{Differential Current Switch Logic}

Differential current switch logic (DCSL) belongs to the class of clocked differential cascode voltage switch logic circuits. DCVS gates operate at all times with both true and complementary signals. Evaluation is carried out with a complementary NMOS transistor tree connected to the true and complementary outputs of the gate. The design constraint for the NMOS transistor tree is that for all possible input combinations at most and at least one path to ground exists from one of the output nodes. Various forms of $D C V S$ gates differ in the circuitry present for generating the output. 


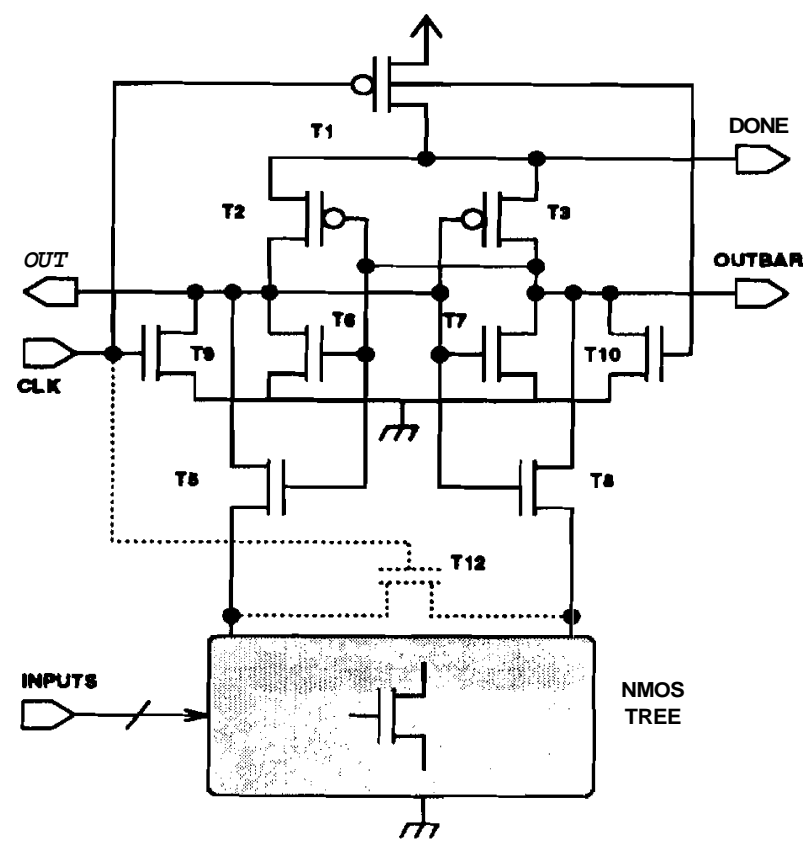

Figure 2: Precharge low DCSL

The structure of the DCSL gate is shown in figure 1. It consists of an $\mathrm{N}$ evaluation tree, a cross coupled inverter pair ( $T 2, T 3, T 6$ and $T \gamma)$ and precharge transistors ( $T 1$ and $T 4$ ). The presence of transistors $T 5$ and $T 8$ is what differentiates this gate from other DCVS logic gates. Operation of the gate starts with CLK low and nodes OUT and OUTBAR being charged high. Gate evaluation begins with stable inputs to the $\mathrm{N}$ tree and $C L K$ going high. CLK high switches T9, T10 and T11 on while OUT and OUT$B A R$ being high ensure that T5,T6,T7 and T8 are switched on. OUT and OUTBAR discharge towards ground through T6, T7 and T10. The discharge of OUT and OUT$B A R$ is not symmetrical because the $\mathrm{N}$ tree assures that one of the outputs say OUT has a stronger path to ground. This causes $O U T$ to fall faster than OUTBAR. The crosscoupled inverter functions as a sense-amplifier and boosts the output voltage differential in the right direction. Once the inverter switch threshold is crossed by OUT, OUTBAR swings high. The low going transition of OUT disconnects the $\mathrm{N}$ tree from OUTBAR by progressively cutting of transistor $T 8$. This is unlike other DCVS circuits where the NMOS pull-down tree is never disconnected from the output. DCVS circuits charge the internal nodes of the NMOS tree up to $V_{C C}-V_{t n}$ ( $V_{C C}$ is the supply voltage $5 v$ volts and $V_{t n}$ is the threshold voltage of the $\mathrm{N}$ device around lvolt), whereas DCSL charges internal nodes to much smaller voltages of the order of lvolt. The gate comes to rest in a state 
with $O U T^{\prime}$ low and OUTBAR high. T12 may be required to prevent charge buildup on internal nodes of the gate.

On completion of evaluation, the fact that the high output (OUTBAR in the previous case) is disconnected from the NMOS tree assures us that further changes in inputs do not propagate to the output. This is unlike most CMOS logic styles, where changes in the inputs of clocked logic, cause DC through currents, or the gate output being destroyed. Strict adherence to the design constraint of building DCVS NMOS trees is no longer required. Gate inputs may cause paths to ground in both halves of the NMOS tree. However assuring that one of the paths has a stronger pull-down than the other, allows the $D C S L$ gate to evaluate its inputs. On completion of evaluation no static: current paths from $V_{C C}$ to $G N D$ exist.

The above discussion considers DCSL logic with outputs precharged high. Figure 2 shows a DCSL gate with outputs which are charged low. The gate imposes a lower clock load and has the advantage of generating completion signals (DONE). Our simulations compare the above gates with similar DCVS design styles, namely Latched Cascode Differential Logic $\left(L C^{\prime} D L\right)$ [3], and Enable Disable Cascode Logic $(E D C L)$ [4]. $L C D L$ has a structure similar to figure 1 while $E D C L$ is similar to to figure 2. DCSL gates in both cases show much bettier rise times, as well as lower power consumption in comparison to comparable DCVS gates. The lower power consumption is primarily due to smaller internal voltage swings in the range of lvolt as compared to 4volts in DCVS designs. All the above logic circuits use a cross-coupled inverter between the output nodes which allows construction of high fan-in gates that are free from charge leakage problems.

$D C S L$ introduces transistors $T 5$ and $T 8$ in order to cut-off the NMOS tree from the output which is driven to a logic high. The strong positive feedback inherent in this structure can upset the operation of the gate if the two halves are not balanced. The circuit will not only amplify differential currents arising from the NMOS tree but also any other current differentials arising from unbalanced output loads. This is especially true for the circuit in figure 2. Balanced layout techniques need to be employed to ensure symmetrical halves for DCSL gates. In cases where the output loads cannot be guaranteed to be the same, buffering close to the gate needs to be employed.

\section{DCSL circuit techniques}

In this section we illustrate circuit techniques using features of DCSL gates. We particularly refer to the self-lockout of inputs which occurs when the gate evaluates. A 


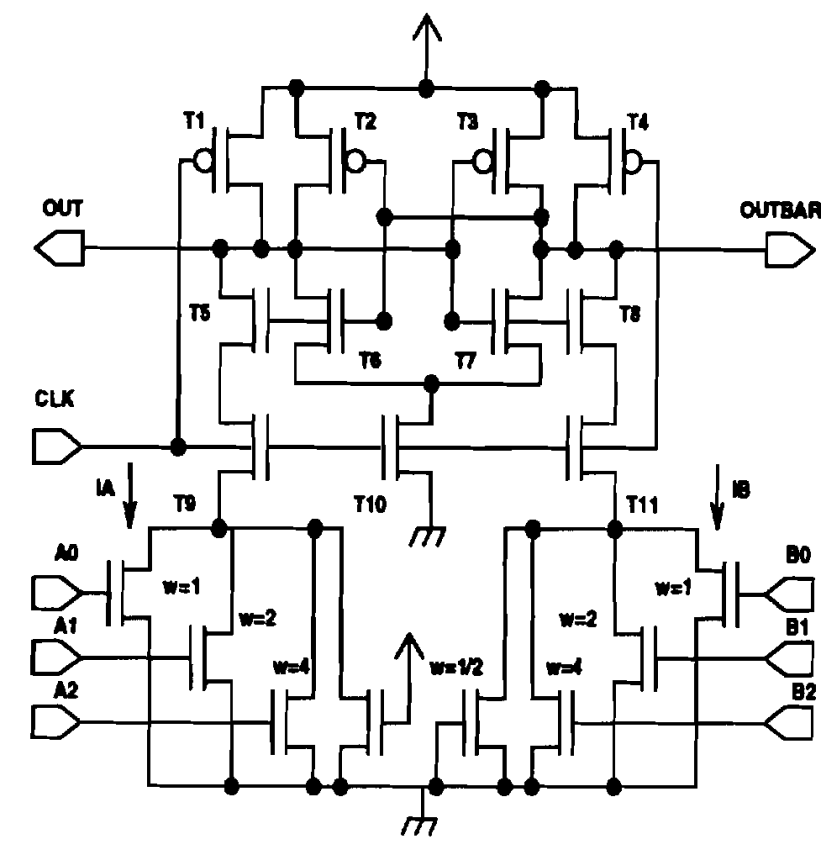

Figure 3: DCSL Magnitude Comparator $(\mathrm{A} \geq \mathrm{B})$

$D C S L$ gate on completion of evaluation does not have any paths from the supply to the ground irrespective of the state of the NMOS tree.

Figure 3 shows a magnitude comparator circuit. The circuit as shown, compares inputs $A$ and $B^{\prime}$ ( 3 bit vectors) and generates outputs indicating whether $A$ is greater than B. This (circuit converts the input say $\boldsymbol{A}$ into an analogue current value (IA in figure 3). The DCSL circuit compares IA and IB and generates an output dependent on the differential of IA and IB. Both halves of the NMOS tree, have paths to ground for all values of $A$ and $\mathrm{B}$ (except $\mathrm{B}=0$ ). The functionality of the gate is correct as long as the accuracy of the conversion of logic inputs to currents is preserved. For the circuit shown this translates into accurate logic high values. Area of the gate would increase drastically with increase in the number of bits being compared, and hence the design as shown may be impractical for more than a few bits. The circuit does illustrate the possibly of using $D C S L$ for the comparison of differential currents.

More important from a system level viewpoint is the design shown in figure 4 . The fact that the transistors in the NMOS tree for DCSL gates need not be turned off completely is utilized in evaluating inputs with a restricted voltage swing. Figure 5 shows the performance of a DCSL gate with inputs which swing partially. Such circuits would be advantageous in driving logic voltages on lines which are heavily loaded. In the circuit 


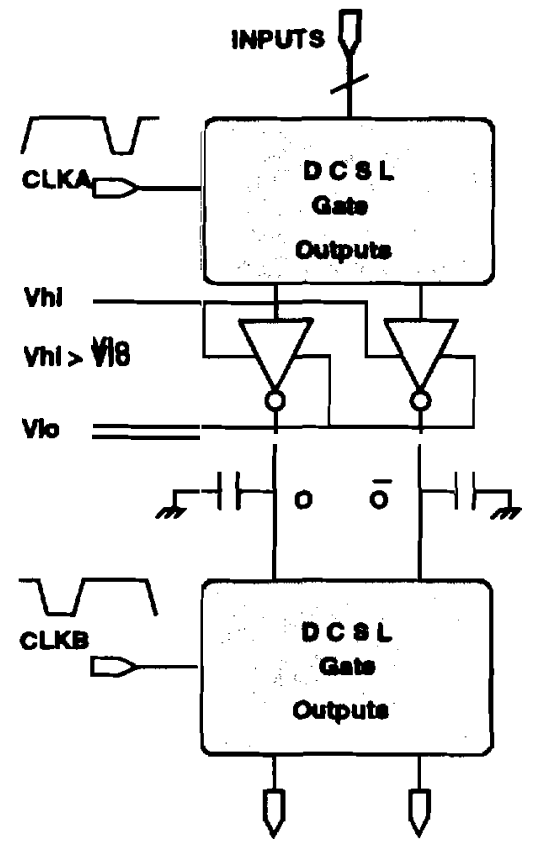

Figure 4: Reduced Voltage Swing $D C S L$

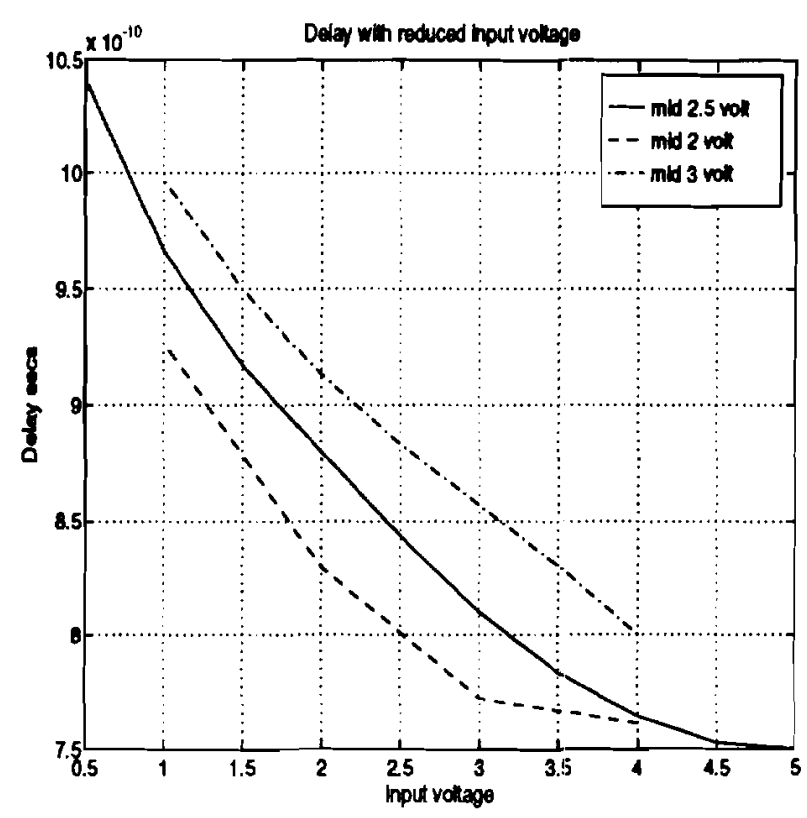

Figure 5: performance with reduced input swings

shown $G N D \leq V_{L O}<V_{H I} \leq V_{C C}$ ( $V_{C C}$ and $G N D$ are the supply and ground voltages). The voltage swing on lines is limited to $V_{H I}-V_{L O}$.

\section{Simulation Results}

SPICE simulations for the circuits described in this paper were carried out using the MOSIS $1.2 \mu$ CMOS process. Results obtained with various tree heights for a parity generator circuit are shown in figure 6 and figure 8. All transistors in the circuits simulated are equally size with width $3 \mu$, except for transistor $T 1$ in figure 2 which is of size $6 \mu$. SPICE simulations are carried out using the level three spice model at $27^{\circ} \mathrm{C}$.

Figure 6 shows the power advantage of the new circuits in comparison to enable-disable DCVS circuits and $L C D L$ gates. This graph shows that energy consumption per transition shows a lower dependence on the number of internal nodes for DCSL gates. DCSL logic favors implementing higher complexity gates. Figure 8 shows that $D C S L$ in general exhibits far superior delay times. This advantage increases as tree height :increases. The circuit shown in figure 2 has the lowest power consumption while the DCSL circuit in figure 1 has the best delay characteristics. The power advantage of DCSL is primar- 


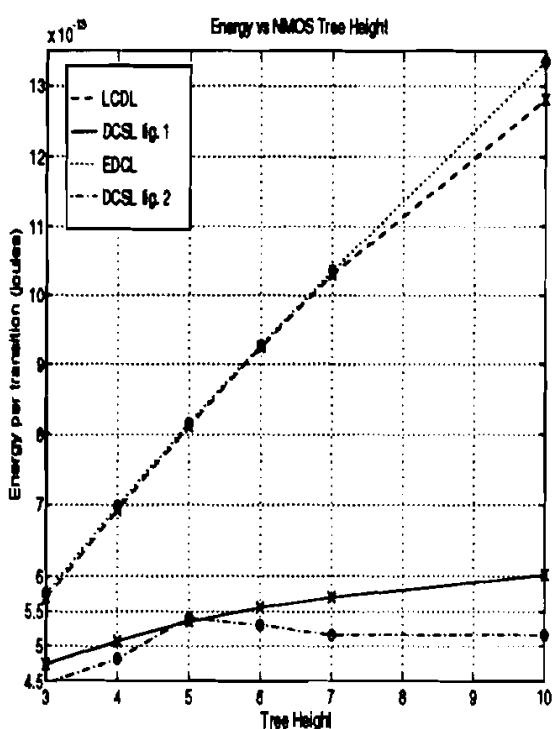

Figure 6: Energy consumption
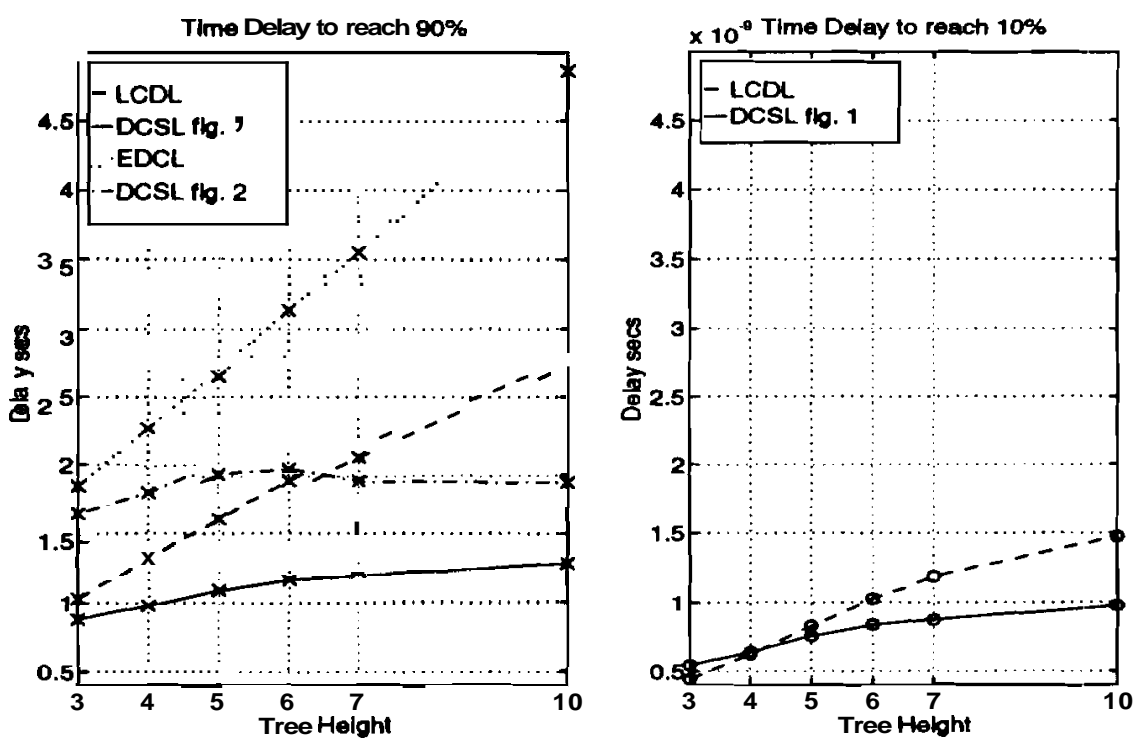

Figure 8: Comparison of gate delays 
ily because of the very low internal voltage swings. Internal node voltages in standard CMOS gates is of the order of 3.5volts. Internal node voltage swings for the DCSL circuit in figure 1 and figure 2 are of the order of lvolt and $\mathbf{0 . 3 v o l t s , ~ r e s p e c t i v e l y . ~}$

A plot of the energy delay product shows the merits of the new circuits. Figure 7 indicates the better energy delay products of DCSL gates. The energy delay products for $D C S L$ gates rises slowly with respect to an increasing NMOS tree height. Delays shown in this graph are the gate delay for outputs to rise to $90 \%$ since this is the largest delay for these circuits.

\section{Summary}

A new clocked logic family, Differential Current Switch Logic (DCSL) is presented in this paper. By reducing internal voltage swings in the gate, we achieve appreciable power savings and better speed as compared to comparable clocked cascode voltage switch circuits. In our opinion the circuit technique used to cut off NMOS evaluation trees is fairly generic and should be applicable to most cascode voltage switch logic families. In fact this paper illustrates two forms of DCSL gates. Insensitivity to inputs after completion of evaluation allows us to reduce the restrictions on the design of the NMOS tree. This allows simpler implementation of logic functions, and the possibility of operating logic with reduced output voltage swings. DCSL circuit techniques can be fully exploited in system designs which impose stringent requirements on power and delay of combinatorial logic bloclks. The ease of generating completion signals, automatic lock-out of inputs, and the low-power of DCSL gates make it a candidate for asynchronous logic: design styles. The drawback of DCSL is that layouts should ensure a symmetrical load on the outputs as well as the NMOS tree, for proper gate operation. 


\section{References}

[1] K. M. Chu, D. L. Pulfrey, "Comparison of CMOS Circuit Techniques: Differential Cascode Voltage Switch Logic versus Conventional Logic", IEEE Journal of Sold State Circuits, vol. 22, no. 4, Aug 1987, pp. 528-532

[2] Wai Lee, Uming Ko and P.T. Balsara, "A Comparative Study on CMOS digital Circuit Fámilies for Low-Power Applications", Proceedings of the Intl. Workshop on Low Power Design 1994, pp. 129-131

[3] Wu Chung-Yu, Cheng Kuo-Hsing, "Latched CMOS differential logic (LCDL) for complex high-speed VLSI", IEEE Journal of Solid State Circuits, vol. 26, no. 9, Sep 1991, pp. 13'24-1328

[4] Lu Shih-Lien L., Milos D Ercegovac, "Evaluation of two-summand adders implemented in ECIDL CMOS differential logic", IEEE Journal of Solid State Circuzts, vol. 26, no. 8, Aug; 1991, pp. 1152-1160 